\title{
Author Correction: Tau binding protein CAPON induces tau aggregation and neurodegeneration
}

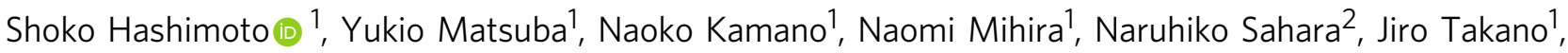 \\ Shin-ichi Muramatsu (1) ${ }^{3,4}$, Takaomi C. Saido (i) ${ }^{1}$ \& Takashi Saito (i) ${ }^{1,5}$
}

Correction to: Nature Communications https://doi.org/10.1038/s41467-019-10278-x, published online 03 June 2019.

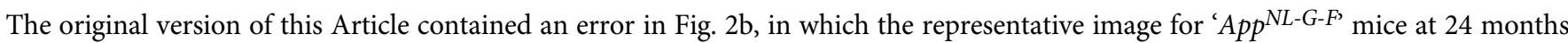
(bottom row, far right) was inadvertently duplicated from the image to its left, the representative image for the 'WT'. This has been corrected in both the PDF and HTML versions of the Article.

Published online: 01 July 2019

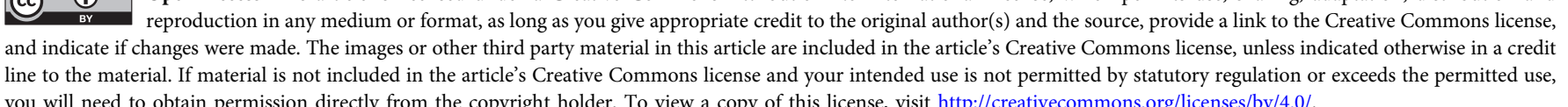
you will need to obtain permission directly from the copyright holder. To view a copy of this license, visit http://creativecommons.org/licenses/by/4.0/.

(C) The Author(s) 2019

\footnotetext{
${ }^{1}$ Laboratory for Proteolytic Neuroscience, RIKEN Center for Brain Science, 2-1 Hirosawa, Wako-City, Saitama 351-0198, Japan. ${ }^{2}$ Department of Functional Brain Imaging Research, National Institute of Radiological Sciences, National Institutes for Quantum and Radiological Science and Technology, 4-9-1 Anagawa, Inage-ku, Chiba-City, Chiba 263-8555, Japan. ${ }^{3}$ Division of Neurology, Jichi Medical University, 3311-1 Yakushiji, Shimotsuke-City, Tochigi 3290498, Japan. ${ }^{4}$ Center for Gene \& Cell Therapy, The Institute of Medical Science, The University of Tokyo, Tokyo 108-8639, Japan. ${ }^{5}$ Department of Neuroscience and Pathobiology, Research Institute of Environmental Medicine, Nagoya University, Nagoya-City, Aichi 464-8601, Japan. Correspondence and requests for materials should be addressed to S.H. (email: shoko.hashimoto@riken.jp) or to T.C.S. (email: saido@brain.riken.jp)

or to T.S. (email: takashi.saito.aa@riken.jp)
} 